\title{
Development of International Guidelines and Education Resources for Cancer-Associated Thrombosis (CAT)
}

Taylor W. Butler, PharmD, BCOP, Carmen Escalante, MD, Carme Font, MD, Cristhiam Mauricio Rojas-Hernandez MD, Thein Oo, MD

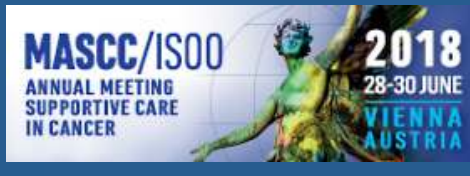

\section{Introduction}

Thrombosis risk is estimated to be as high as $18 \%$ in cancer patients and a source of morbidity and mortality. The hemostasis study group organized members to develop clinical practice guidelines for cancer-associated thrombosis (CAT). The 2017 MASCC/ISOO workshop hosted by the hemostasis/education study group revealed that further education materials might be indicated to improve patient understanding.

\section{Objectives}

To review the recent literature to determine recommendations on CAT with an initial focus on inpatient practice primary prevention. Of note, information will also be collected to develop patient friendly educational tools.

\section{Methods}

The co-chairs met with MASCC members periodically through teleconference to discuss project development. We adapted literature search methods similar to previous clinical practice guidelines published by MASCC/ISOO. The co-chairs conducted the initial literature search using MEDLINE and the Cochrane database and eliminated studies if the abstract revealed exclusion criteria. All other studies were pulled and divided to each participant. Grading and recommendations will follow previous guidelines by MASCC/ISOO.

\section{Results}

1589 studies were reviewed during the literature search. 1336 were eliminated based on the abstract. The following 253 studies have been assigned to each member and the full article will be reviewed for inclusion/exclusion criteria and grading, if applicable.

$$
\begin{gathered}
1589 \text { abstracts reviewed } \\
n=426 \text { Cochrane } \\
n=1163 \text { MEDLINE }
\end{gathered}
$$

1336

eliminated

253 for full

text review

\section{Conclusion}

Each study participant will review at least 21 studies with the goal of developing guidelines and educational tools for inpatient CAT prophylaxis. The target date is to present to the participants of the Hemostasis study group in Vienna and to the executive leaders of MASCC/ISOO in 2019. 